\title{
Locoregional Treatment for De Novo Metastatic Nasopharyngeal Carcinoma
}

\author{
(D) Yavuz DiZDAR, (1) Kübra ÖZKAYA TORAMAN, (1) Musa ALTUN \\ Department of Radiation Oncology, İstanbul University Faculty of Medicine, İstanbul-Turkey
}

\begin{abstract}
OBJECTIVE
Optimal management of de novo metastatic nasopharyngeal carcinoma (NPC) is debatable. The aim of this study is to evaluate the patient characteristics, the impact of locoregional treatment on disease control and overall survival (OS) and to analyze the factors that correlate with the outcome of patients with de novo metastatic NPC patients treated between 2000 and 2018.
\end{abstract}

\section{METHODS}

Among 589 NPC patients referred to our clinic in the past 18 years, the cases of 36 de novo metastatic NPC patients who received radical locoregional radiotherapy (LR-RT) were analyzed retrospectively. After excluding one patient who had previously received chemotherapy for 12 courses in another clinic, the remaining 35 patients were analyzed in terms of population characteristics, OS, and possible confounding factors.

\section{RESULTS}

Seven of 35 patients were under the age of 16 . The histology was World Health Organization (WHO) type $2-3$ in $94.3 \%$. All but two patients received 3-6 cycles of induction chemotherapy. The median dose of LR RT was 70 Gy. The median follow-up time was 25 months. Two and 4 year OS rate was $51 \%$ and $34 \%$, respectively. Univariate analysis showed no significant effects of age $(>6, \leq 40)$, gender, oligometastatic disease, the existence of liver metastasis, or RT dose on the OS.

\section{CONCLUSION}

De novo metastatic NPC patients had highly prolonged survival with the use of LR-RT and this treatment approach should be validated by further multi-centric clinical studies.

Keywords: De novo; distant metastasis; nasopharyngeal carcinoma; outcomes; radiation therapy. Copyright $\odot$ 2021, Turkish Society for Radiation Oncology

\section{Introduction}

Nasopharyngeal carcinoma (NPC) differs from other head and neck carcinomas by its specific geographic and ethnic distribution, its association with EpsteinBarr virus (EBV) infection, and predisposition of distant metastases. [1]

NPC is also chemotherapy and radiotherapy (RT) sensitive disease with distinct demographic, clinical, staging, and treatment options as compared to non-nasopharyngeal head and neck cancer.[2] RT is the fundamental treatment modality and concurrent chemo$\mathrm{RT}$ is recommended for locoregionally advanced NPC according to the National Comprehensive Cancer Network (NCCN) Guidelines.[3] However, distant metastasis remains a key challenge.

Both synchronous and metachronous distant metastases are more common among NPC compared to other 
head and neck cancers. [4-6] For synchronous distant metastasis of NPC, this rate ranges between 6 and 15\%. $[7,8]$ Most of the current oncological treatment guidelines suggest chemotherapy as the only treatment option for metastatic disease in NPC. $[9,10]$ This might be seen as a reflection of the old perspective which limits RT role with cancer palliation, mostly for symptom control. Only the NCCN guidelines regard post-chemotherapy locoregional RT (LR-RT) as a treatment option without any suggestion on treatment or follow-up. [3]

In recent years, the interest in LR-RT of primary tumors with distant metastases has increased. The survival benefit of local treatment directed at all metastasis in oligometastatic disease has been demonstrated in an early randomized study.[11] The effectiveness of local therapies to the primary tumor in metastatic disease is evolving for some specific cancer types.

According to the "seed and soil" hypothesis, the soluble growth factors secreted from the primary tumor causes the clustering of hematopoietic progenitor cells and macrophages, creating an environment conducive to the spread of malignant clones and the formation of metastasis.[12,13] This emphasizes the importance of the local tumor stage and the possible contribution of local therapies to survival in patients with distant metastases.[14] In accordance with this thesis, the survival benefit of local therapy in metastatic renal cell cancers and transitional cell bladder cancer has been demonstrated. $[14,15]$ Similarly, the role of radical LRRT in metastatic NPC has been investigated in several retrospective studies[16-19] and in a very recent a prospective randomized trial.[20] They conclude LRRT infer a positive effect on OS.

Following induction chemotherapy, we deliver radical LR RT in de novo metastatic NPC (unless obvious progression under chemotherapy) for more than two decades due to the survival advantage observed in our clinical practice. We aimed to share our retrospectively evaluated data of patients with nasopharyngeal cancer who had distant metastases at the time of diagnosis and were treated with LR RT in terms of clinical features and survival.

\section{Materials and Methods}

589 cases of patients who had biopsy-proven nasopharyngeal cancer referred to our clinic between 2000 and 2018 were assessed and 36 patients who had distant metastases at diagnosis and also received radical dose $\mathrm{RT}$ to the head and neck region were identified. After excluding one patient who had previously received chemotherapy for 12 courses in another clinic, the remaining 35 patients were included in the analysis.

\section{Pretreatment Evaluation}

All patients received pre-treatment evaluation consisting of a complete history and physical examination, endoscopic examination, complete blood counts, blood chemistries, computed tomography (CT), or magnetic resonance imaging (MRI) of the nasopharynx and neck.

Until 2006, all patients were screened for distant metastases, using chest radiography, Technetium-99m bone scintigraphy, and abdominal ultrasonography (USG). After 2006, $\left({ }^{18} \mathrm{~F}\right.$-fluorodeoxyglucose positron emission tomography-CT [PET-CT]) superseded these methods. Tumors were staged according to the $7^{\text {th }}$ edition of the American Joint Cancer Committee (AJCC) TNM staging system.

\section{Treatment}

De novo metastatic NPC patients eligible for combined modality chemotherapy received 3-6 courses of 33 of the 35 patients. The chemotherapy responses were evaluated by CT or MRI. The metastases were screened by abdominal USG, chest radiography, bone scintigraphy, or PET-CT. The patients with partial/ complete chemotherapy response were assessed for RT. A total of 60-74 Gy of RT were administered with daily fractions of 1.8-2 Gy. In patients under the age of 16, the RT dose was reduced (60-63 Gy) and concurrent chemotherapy was not used. For the patients older than 16 years of age, the decisions for the concurrent chemotherapy were made on a patient basis. Side effects during RT were monitored weekly.

\section{Follow-up Evaluations}

The periods for follow-up exams were 1 month- 3 months for the first 2 years after RT, 4-6 months for the $3^{\text {rd }}, 4^{\text {th }}$, and $5^{\text {th }}$ years and annually thereafter. Complete blood count, blood biochemistry, and endoscopic examination were performed at each control. Head and neck region evaluation was performed annually by CT or MRI. PETCT evaluation was held 3 months after the end of LR treatment and else when there is clinical indication.

\section{Statistical Analysis}

OS was calculated from the date of diagnosis to the day of death for any reason or date of the last follow up. Survival was estimated by the Kaplan-Meier method. Potential prognostic factors for OS, including age, sex, radiation dose, chemotherapy, and site of metastases, number of metastatic disease, and liver metastasis were 
evaluated using log-rank comparisons. $\mathrm{P}<0.05$ was considered statistically significant. All statistical analyses were performed using SPSS version 26.

\section{Results}

The median age of the patients was 49 (9-85 years) and $85.7 \%(n=30)$ of them were male. Overwhelming majority of the patients had either undifferentiated carcinoma $65.7 \%(n=23)$ or non-keratinized carcinoma $28.6 \%(\mathrm{n}=10)$ only $2(5.7 \%)$ of the 35 patients and keratinized carcinoma. PET-CT was used in $82.9 \%$ $(n=29)$ of the patients at diagnosis. Eight (22.9) patients had a single metastasis. Furthermore, $17.1 \%$ of patients were oligometastatic. The remaining $60 \%$ was multiple metastatic. Thirteen (37.1\%) of the patients had multiple organ metastases. Bone metastasis was present in $71.4 \%$ of patients, liver metastasis in $22.9 \%$, and lung metastasis in $20 \%$. Three patients had mediastinal lymph node metastasis, three patients had bone marrow involvement, one patient had axillary lymph nodes, one patient had adrenal, and one patient had para-aortic lymph node metastasis (Table 1).

Except for two patients with insufficient renal function, all patients first received 3-6 cycles of chemotherapy. While the median dose of RT was $7000 \mathrm{cGy}$ (6000-7400), a reduced dose of 6000-63.00 cGy RT was applied to patient's $\leq 16$ years of age for treatment (Table 2). Eighteen patients received RT concurrently with chemotherapy (one patient, carboplatin; 17 patients, cisplatin). Palliative bone irradiation was performed in 19 patients after LR RT, and one patient received radioembolization for liver metastasis (Table 2).

The median follow-up time was 25 months (5-196 months). During the follow-up period, 24 patients died, one surviving patient with active disease continues to be treated with chemotherapy. Ten patients were on follow-up without disease (48-196 months). Six of the ten patients are alive for more than 5 years without disease. 4-year-survival was calculated as $34 \%$ (Fig. 1). In univariate analysis, none of the factors (age $\leq 40$ years, gender, oligometastatic disease, presence of liver metastasis, and RT dose) effected survival.

\section{Discussion}

It has been reported that $6-15 \%$ of NPC patients are diagnosed with de novo metastatic cancer before any treatment has begun. $[7,8]$ By the development of PET$\mathrm{CT}$ at the end of the 1990s, its sensitivity and specificity in cancer staging have begun to be investigated. In their

\begin{tabular}{|c|c|c|}
\hline \multirow[t]{2}{*}{ Table 1} & \multicolumn{2}{|c|}{ Patient and treatment characteristic } \\
\hline & $\mathbf{n}$ & $\%$ \\
\hline \multicolumn{3}{|l|}{ Gender } \\
\hline Male & 30 & 85.70 \\
\hline Female & 5 & 14.30 \\
\hline \multicolumn{3}{|c|}{ Histopathology } \\
\hline WHO1 & 2 & 5.70 \\
\hline WHO2 & 10 & 28.60 \\
\hline WHO3 & 23 & 65.70 \\
\hline \multicolumn{3}{|l|}{ İnitial PET-CT } \\
\hline Yes & 29 & 82.90 \\
\hline No & 6 & 17.10 \\
\hline \multicolumn{3}{|l|}{ T stage } \\
\hline $\mathrm{T} 1$ & 2 & 5.70 \\
\hline $\mathrm{T} 2$ & 20 & 57.10 \\
\hline T3 & 5 & 14.30 \\
\hline $\mathrm{T} 4$ & 8 & 22.90 \\
\hline \multicolumn{3}{|l|}{$\mathrm{N}$ stage } \\
\hline No & 1 & 2.90 \\
\hline N2 & 18 & 51.40 \\
\hline N3 & 16 & 45.70 \\
\hline \multicolumn{3}{|c|}{ No. of metastatic lesions } \\
\hline Single & 8 & 22.90 \\
\hline Oligo & 6 & 17.10 \\
\hline Multiple & 21 & 60.00 \\
\hline \multicolumn{3}{|c|}{ No. of metastatic organs } \\
\hline Single & 22 & 62.90 \\
\hline Multiple & 13 & 37.10 \\
\hline \multicolumn{3}{|c|}{ Liver metastasis } \\
\hline Yes & 8 & 22.90 \\
\hline No & 27 & 77.10 \\
\hline \multicolumn{3}{|c|}{ Induction chemotherapy } \\
\hline Yes & 33 & 94.30 \\
\hline No & 2 & 5.70 \\
\hline \multicolumn{3}{|c|}{ Concurrent chemotherapy } \\
\hline Yes & 18 & 51.40 \\
\hline No & 17 & 48.60 \\
\hline
\end{tabular}

WHO: World Health Organization; PET: Positron emission tomography; T stage: Primary tumor staging; N stage: Node staging

trial comparing four different staging methods $(\mathrm{n}=78)$, Chua et al.[21] found PET-CT superior in the terms of sensitivity, specificity, and accuracy to the conventional methods (chest radiography, abdominal USG, and bone scan) (Also the conventional methods performed poorly in this trial, missing four of six metastases). $\mathrm{Ng}$ et al.[22] reported a false positivity rate of $18 \%$ with PET-CT for their prospective trial $(n=115)$. Tang et al.[8] $(n=583)$ showed that PET-CT detects more distant metastases than conventional staging in patients with NPC and the largest benefit in terms of cost and patient management was observed in the subgroup 


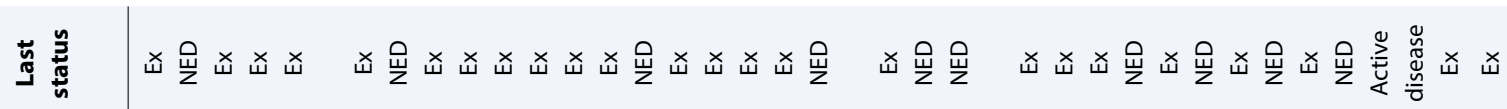

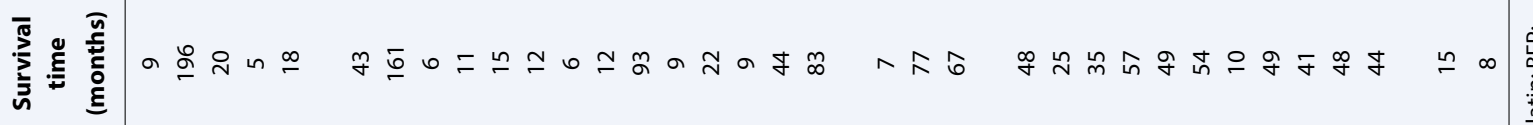

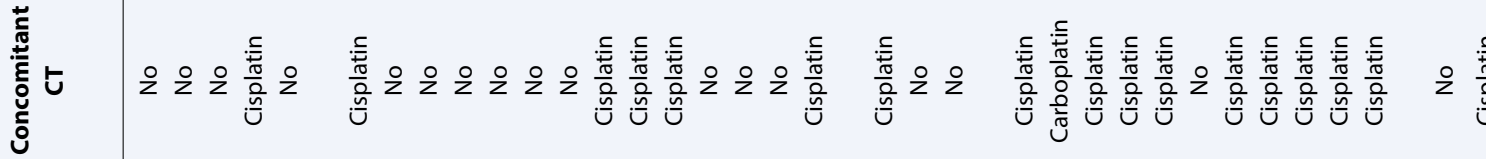

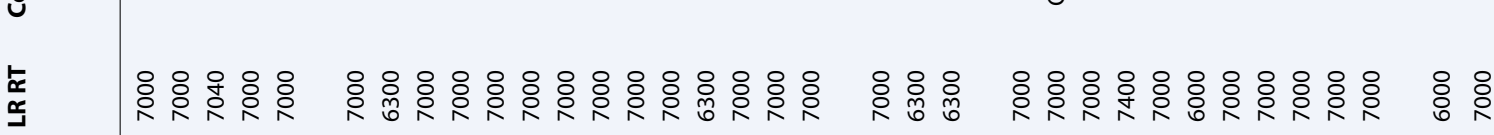

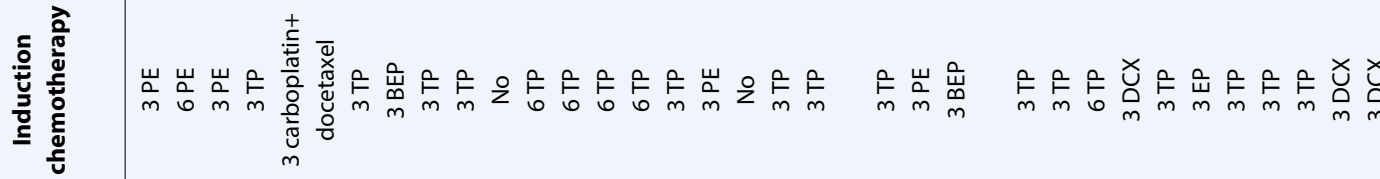
离离䔍

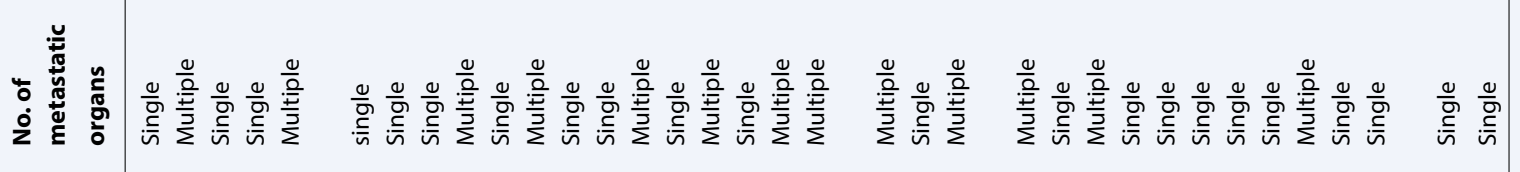

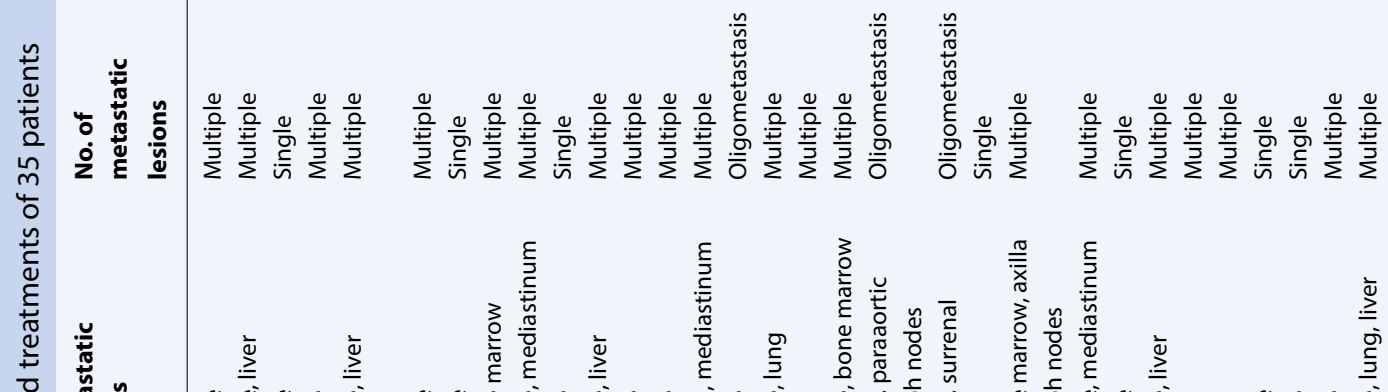

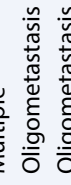
넌 


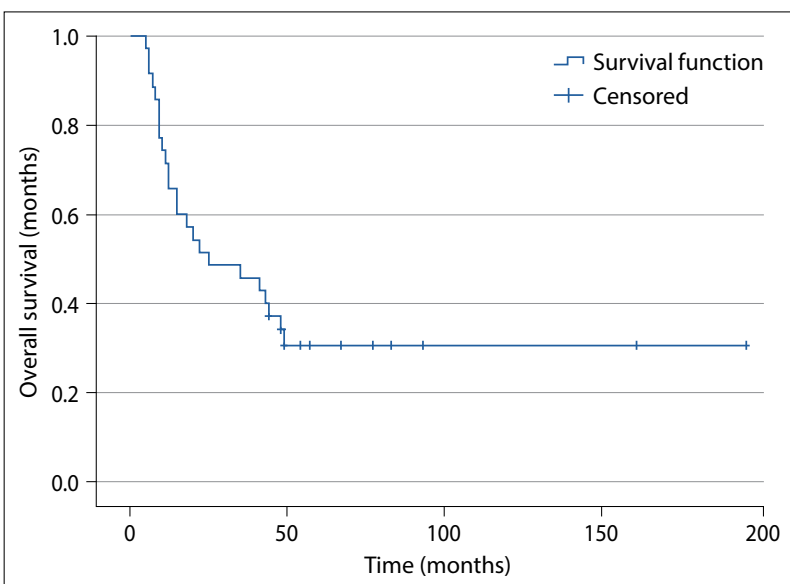

Fig. 1. Overall survival of all patients.

with N2-3 disease and EBV DNA $\geq 4000$ copies/mL. In our clinic, we have been staging the NPC patients using PET-CT, irrespective of their local stage since 2006. Six of the patients (17\%) analyzed in this study were staged previously with conventional methods. All patients in the study were stage N2-3 in regional staging, except one patient with $\mathrm{T} 4 \mathrm{~N} 0$ disease.

In the last 18 years, 589 NPC patients have been referred to our clinic, $6.1 \%$ of them had distant metastases at the time of diagnosis. In this current study of 35 de novo metastatic NPC patients with a median follow-up time of 25 months, the 4-year-survival was $34 \%$ and no significant prognostic factor on survival could be identified.

Local therapy has been used for metastatic disease with the intent of reducing primary tumor burden, relieving symptoms, or propagation of metastases. Some cancer studies have demonstrated that intensive local therapy could prolong overall survival (OS) in untreated de novo metastatic cancer patients. $[14,15,23,24]$ This concept of LR treatment is supported by a randomized clinical trial reporting the OS benefit of high dose RT to the primary tumor (STAMPEDE).[25] The number of clinical studies researching the effect of LR RT in de novo metastatic NPC is limited. It should, however, be cleared since treatment of de novo metastatic NPC patients must consider the control of primary tumors, which is different from metastatic NPC after treatment. Several retrospective analyses suggested that additional LR RT could improve survival of these patients in addition to palliative chemotherapy.[16-19] In accordance with these emerging data, the NCCN Guidelines recommend concurrent chemoradiation as an option in de novo metastatic NPC.
NPC usually metastasize to bones, lungs, and liver. $[2,26]$ Among them, the primary metastasis site is the bones. The solitary bone metastasis is alleged to be related to a better prognosis than the others. [26] The liver, the third common metastasis site after the lungs, has the worst survival rates according to Zou et al. study.[19] They separated the M stage into three subgroups, according to the number of metastatic lesions and the existence of liver metastasis in their classification. While the oligometastatic disease subgroup had the best survival rates, the subgroup with the liver metastasis, which was named M1c, had the worst. In their study using five different prognostic factors (age, $\mathrm{N}$ stage, number of metastases, organ involvement, and EBV DNA levels), Sun et al.[27] found the existence of multiple metastases and liver involvements, negative prognostic factors. In our serial, there were eight patients with liver metastases (one patient under 16 years of age, one single-metastatic-patient, and two oligometastatic-patients) and three of them are alive with no evidence of disease for 77, 83 and 196 months.

In their retrospective data Lin et al.[16] evaluated 105 patients with de novo metastatic NPC and stated better survival rates for single metastatic patients treated with RT doses higher than 65 Gy. Among the eight single-metastatic-patients in our serial, three of them are alive, continuing their life disease-free.

In 2020, You et al.[20] published a two-armed, Phase III randomized trial, investigating the effectiveness of LR RT in de novo metastatic NPC patients with partial or complete response to three cycles of cisplatin, and 5-fluorouracil (PF) treatment. While the control group (who had only taken chemo) had a 2-year-survival rate of $54 \%$, and the 2-year-survival rate of the CT+RT group was $76 \%(\mathrm{p}=0.004)$. In this trial, $30.9 \%$ of patients had one or two metastases, and this group had longer OS. It is the first and sole Phase III trial showing the contribution of LRRT in de novo metastatic NPC patients with good response to PF chemotherapy. The exclusion of the unresponsive patients to PF chemotherapy limits the generalizability of the results, the results are still important since they point out the value of LR RT in de novo metastatic NPC treatment. In this trial, the RT dose following PF was $7000 \mathrm{cGy}$ and the irradiated RT volumes were designated according to pre-chemotherapy imaging. In the same issue with article invited commentators suggested limiting irradiation with postchemotherapy volumes and the dose of $60 \mathrm{~Gy}$ in the patients with complete response.[28] However, yet there is no convincing proof for the dose decrement in this group with a long survival (You et al., 2-year-survival 
$>50 \%$ ). In our clinical practice, we use pre-chemotherapy imaging and apply $70 \mathrm{~Gy}$ for adult patients.

\section{Limitations of the Study}

This is a retrospective study with a limited sample size. Although it reflects the two decades of experience of a single center in de novo metastatic disease treated with a considerably homogenous program the patient group consisted of various age groups (9-85), had different chemotherapy regimens, their number and sites of metastases varied. This complicates the investigation of survival related factors.

\section{Conclusion}

The LR treatment in de novo metastatic diseases is gaining prominence since the related patient group can have long survival depending on the count of their metastases and organ involvement. De novo metastatic NPC patients had highly prolonged survival with the use of LR $\mathrm{RT}$ and this treatment approach should be validated by further multi-centric clinical studies. In our clinic, postchemotherapy LR RT constitutes the primary treatment option for de novo metastatic NPC.

Peer-review: Externally peer-reviewed.

Conflict of Interest: The authors have no conflicts of interest to declare.

Ethics Committee Approval: The study was approved by the Local Ethics Committee of Istanbul University Oncology Institute (No: 70973125-604.01.01, Date: 09/12/2020).

Financial Support: The authors declared that this study has received no financial support.

Authorship contributions: Concept - M.A.; Design Y.D., K.Ö.T.; Supervision - M.A.; Funding - None; Materials - K.Ö.T., Y.D.; Data collection and/or processing - K.Ö.T., Y.D.; Data analysis and/or interpretation - K.Ö.T., Y.D.; Literature search - K.Ö.T., Y.D.; Writing - K.Ö.T., Y.D., MA.; Critical review - M.A.

\section{References}

1. Lee A, Lin J, Ng W. Current management of nasopharyngeal cancer. Semin Radiat Oncol 2012;22(3):23344.

2. Fandi A, Altun M, Azli N, Armand JP, Cvitkovic E. Nasopharyngeal cancer: epidemiology, staging, and treatment. Semin Oncol 1994;21(3):382-97.

3. National Comprehensive Cancer Network. Available at: https://www.nccn.org/professionals/physician_gls/ pdf/head-and-neck.pdf. Accessed Mar 10, 2021.
4. Chen WZ, Zhou DL, Luo KS. Long-term observation after radiotherapy for nasopharyngeal carcinoma (NPC). Int J Radiat Oncol Biol Phys 1989;16(2):311-4.

5. Frezza G, Barbieri E, Emiliani E, Silvano M, Babini L. Patterns of failure in nasopharyngeal cancer treated with megavoltage irradiation. Radiother Oncol 1986;5(4):287-94.

6. Ho JH. An epidemiologic and clinical study of nasopharyngeal carcinoma. Int J Radiat Oncol Biol Phys 1978;4(3-4):182-98.

7. Lee AW, Poon YF, Foo W, Law SC, Cheung FK, Chan DK, et al. Retrospective analysis of 5037 patients with nasopharyngeal carcinoma treated during 1976-1985: overall survival and patterns of failure. Int J Radiat Oncol Biol Phys 1992;23(2):261-70.

8. Tang LQ, Chen QY, Fan W, Liu H, Zhang L, Guo L, et al. Prospective study of tailoring whole-body dualmodality [18f] fluorodeoxyglucose positron emission tomography/computed tomography with plasma Epstein-Barr virus DNA for detecting distant metastasis in endemic nasopharyngeal carcinoma at initial staging. J Clin Oncol 2013;31(23):2861-9.

9. Chan AT, Grégoire V, Lefebvre JL, Licitra L, Hui EP, Leung SF, et al; EHNS-ESMO-ESTRO Guidelines Working Group. Nasopharyngeal cancer: EHNS-ESMOESTRO Clinical Practice Guidelines for diagnosis, treatment and follow-up. Ann Oncol 2012;23(Suppl 7):vii83-5.

10. Lang J, Hu C, Lu T, Pan J, Lin T. Chinese expert consensus on diagnosis and treatment of nasopharyngeal carcinoma: evidence from current practice and future perspectives. Cancer Manag Res 2019;11:6365-76.

11. Palma DA, Olson R, Harrow S, Gaede S, Louie AV, Haasbeek C, et al. Stereotactic ablative radiotherapy versus standard of care palliative treatment in patients with oligometastatic cancers (SABR-COMET): a randomised, phase 2, open-label trial. Lancet 2019;393(10185):2051-8.

12. Kaplan RN, Psaila B, Lyden D. Bone marrow cells in the "pre-metastatic niche": within bone and beyond. Cancer Metastasis Rev 2006;25(4):521-9.

13. Hiratsuka S, Watanabe A, Aburatani H, Maru Y. Tumour-mediated upregulation of chemoattractants and recruitment of myeloid cells predetermines lung metastasis. Nat Cell Biol 2006;8(12):1369-75.

14. Seisen T, Sun M, Leow JJ, Preston MA, Cole AP, GelpiHammerschmidt F, et al. Efficacy of high-intensity local treatment for metastatic urothelial carcinoma of the bladder: a propensity score-weighted analysis from the national cancer data base. J Clin Oncol 2016;34(29):3529-36.

15. Flanigan RC, Salmon SE, Blumenstein BA, Bearman SI, Roy V, McGrath PC, et al. Nephrectomy followed by interferon alfa- $2 b$ compared with interferon $a l f a-2 b$ 
alone for metastatic renal-cell cancer. N Engl J Med 2001;345(23):1655-9.

16. Lin S, Tham IW, Pan J, Han L, Chen Q, Lu JJ. Combined high-dose radiation therapy and systemic chemotherapy improves survival in patients with newly diagnosed metastatic nasopharyngeal cancer. Am J Clin Oncol 2012;35(5):474-9.

17. Chen MY, Jiang R, Guo L, Zou X, Liu Q, Sun R, et al. Locoregional radiotherapy in patients with distant metastases of nasopharyngeal carcinoma at diagnosis. Chin J Cancer 2013;32(11):604-13.

18. Rusthoven CG, Lanning RM, Jones BL, Amini A, Koshy M, Sher DJ, et al. Metastatic nasopharyngeal carcinoma: patterns of care and survival for patients receiving chemotherapy with and without local radiotherapy. Radiother Oncol 2017;124(1):139-146.

19.Zou X, You R, Liu H, He YX, Xie GF, Xie ZH, et al. Establishment and validation of M1 stage subdivisions for de novo metastatic nasopharyngeal carcinoma to better predict prognosis and guide treatment. Eur J Cancer 2017;77:117-26.

20. You R, Liu YP, Huang PY, Zou X, Sun R, He YX, et al. Efficacy and safety of locoregional radiotherapy with chemotherapy vs chemotherapy alone in de novo metastatic nasopharyngeal carcinoma: a multicenter phase 3 randomized clinical trial. JAMA Oncol 2020;6(9):1345-52.

21. Chua ML, Ong SC, Wee JT, Ng DC, Gao F, Tan TW, et al. Comparison of 4 modalities for distant metastasis staging in endemic nasopharyngeal carcinoma. Head Neck 2009;31(3):346-54.

22. Ng SH, Chan SC, Yen TC, Chang J, Liao CT, Ko SF, et al. Staging of untreated nasopharyngeal carcinoma with PET/CT: comparison with conventional imaging work-up. Eur J Nucl Med Mol Imaging 2009;36(1):1222.

23. Mickisch GH, Garin A, van Poppel H, de Prijck L, Sylvester R, et al. radical nephrectomy plus interferonalfa-based immunotherapy compared with interferon alfa alone in metastatic renal-cell carcinoma: a randomised trial. Lancet 2001;358(9286):966-70.

24. Gomez DR, Blumenschein GR Jr., Lee JJ, Hernandez $\mathrm{M}$, Ye R, Camidge DR, et al. Local consolidative therapy versus maintenance therapy or observation for patients with oligometastatic non-small-cell lung cancer without progression after first-line systemic therapy: a multicentre, randomised, controlled, phase 2 study. Lancet Oncol 2016;17(12):1672-82.

25. Parker CC, James ND, Brawley CD, Clarke NW, Hoyle AP, Ali A, et al; evaluation of drug efficacy (STAMPEDE) investigators. Radiotherapy to the primary tumour for newly diagnosed, metastatic prostate cancer (STAMPEDE): a randomised controlled phase 3 trial. Lancet 2018;392(10162):2353-66.

26. Kumar A, Kumar H, Dhanushkodi M, Ganesharajah S. Metastatic nasopharyngeal carcinoma: outcome from Cancer Institute, Chennai. Indian J Med Paediatr Oncol 2020;41:198-201.

27. Sun XS, Liang YJ, Liu SL, Li XY, Chen QY, Guo SS, et al. Establishment and validation of a nomogram for predicting survival in patients with de novo metastatic nasopharyngeal carcinoma. Oral Oncol 2019;94:73-9.

28. Riaz N, Sherman E, Lee N. The importance of locoregional therapy in metastatic nasopharyngeal cancer. JAMA Oncol 2020;6(9):1353-4. 